\title{
Use of Maximum Surgical Order Schedule (MSBOS) among pediatric patients to optimize blood utilization
}

\author{
Johann Paulo S. Guzman *1D, Leandro L. Resurreccion III and Maria Beatriz P. Gepte
}

\begin{abstract}
Background: Evaluating blood ordering and subsequent development of a blood ordering schedule can decrease over-ordering of blood among pediatric surgical patients. The objective is to assess our practice of blood utilization using various blood utilization indices and calculate the Maximal Surgical Blood Order Schedule (MSBOS). This is a cross-sectional study for 3 years that included patients ( $\leq 18$ years old) who underwent major surgical procedures in the Philippine Children's Medical Center. Data included type of surgery, age and sex, number of units crossmatched, number of units transfused, and timing of transfusion. Indices were calculated.

Results: This study revealed that the utilization rate was only $39.1 \%$ of blood among a total of 5314 cases done. The indices were analyzed according to different procedures among different age groups. Procedures for head and neck, colorectal, ostomy, solid tumors, genitourinary abnormalities, upper gastrointestinal, and appendectomy had $\mathrm{CT}$ (crossmatched to transfusion) ratio exceeding 2.0, indicating inappropriate blood ordering. Major abdominal, major thoracic, and hepatobiliary surgery all have $C T$ ratio $<2.0$. MSBOS was calculated, and a requisition of 1 unit of blood among patients undergoing these procedures is suggested.
\end{abstract}

Conclusions: The MSBOS is largely utilized to the adult population but its creation can also be utilized among pediatric patients. Our data shows that in majority of elective pediatric surgical procedures, routine crossmatch is not necessary which is proven by our high underutilization of requested blood products.

Keywords: Maximum Surgical Blood Order Schedule (MSBOS), CT ratio, Blood indices

\section{Background}

It is observed that there is an excessive pre-operative over-ordering of blood products among elective pediatric elective patients leading to increasing costs and waste of limited supply of blood. A blood ordering system should be created so that excessive or even unnecessary blood ordering is prevented. Such ordering system may be created if we study the indices used in blood ordering for each specific surgical case; amount of blood ordered for that case and the blood eventually transfused in that case, so that we can tailor blood ordering to a specific surgical patient.

There have been suggestions by clinicians to establish a structured ordering system to improve blood utilization. The main objective of creating a MSBOS is to reduce

\footnotetext{
* Correspondence: yooohannn@yahoo.com

Division Of Pediatric Surgery, Philippine Childrens Medical Center, Quezon City 1100, Philippines
}

胞 Springer Open

(c) The Author(s). 2019 Open Access This article is distributed under the terms of the Creative Commons Attribution 4.0 International License (http://creativecommons.org/licenses/by/4.0/), which permits unrestricted use, distribution, and reproduction in any medium, provided you give appropriate credit to the original author(s) and the source, provide a link to the Creative Commons license, and indicate if changes were made. large quantities of crossmatched blood for surgical patients of which little is utilized creates an artificial shortage in the reserves, wastes valuable technical time, and squanders expensive reagent [2]. Blood banks face an everincreasing demand for blood and its components. It has been reported that only 30\% of crossmatched blood is used in elective surgery [3]. In a report by Mwambungu et al. in 2015 , only $64 \%$ of crossmatched blood was utilized [4]. The review of literature reports that underutilization of blood products or unnecessary blood requisition is still common in most hospitals.

Common variations in rates of transfusion may be due to many factors, including differing opinions in the threshold level of hemoglobin below which a patient needs blood transfusion, differences in surgical and anesthetic techniques, cancelation of cases, differences in case mix, pre-operative anemia, and a lack of availability 
of transfusion protocols. Unnecessary ordering of blood for surgical patients can be reduced without having any detrimental effect on the safety of the patient. The use of blood conservation policies such as the MSBOS has succeeded in limiting unnecessary transfusion practices. MSBOS (1.5× transfusion index) estimates the amount of blood that will be needed for the individual procedure. This is the criterion developed from usage statistics providing a figure for the number of units to be crossmatched for any surgical procedure $[1,5]$.

Pre-operative blood ordering protocols generally do not contemplate the differences between patients with high and low risks of bleeding. Probably, it is important to assess the influence of the surgical technique itself instead of the duration. In two studies of adult elective patients undergoing minimally invasive versus open colon surgery, there is a difference in intra-operative transfusion rates [6,7]. This may hold true among pediatric patients that blood transfusion protocols should be guided by the procedure. Although, some physicians often argue that blood products should be ready during surgery in case a need arises to prevent complications due to the low blood volume in pediatric patients. This ideology mitigates rising hospital costs in the event of unnecessary blood pre-ordering. But such ideology wastes precious hospital resources, and thus, a formulation of blood transfusion protocols in elective surgery patients should be created and be widely accepted among practitioners.

\section{Methods}

\section{Research design and description of study}

This is a 3-year cross-sectional study that included pediatric surgical patients ( $\leq 18$ years old) that underwent major surgical procedures in the Division of Pediatric Surgery of the Philippine Children's Medical Center from January 2014 up to December 2016. Patient's data were collected from the Institution's Blood Center transfusion data from an electronic database and our monthly surgical census database. Data collected included type of surgery, age and sex of the patient, preoperative investigations including the number of units crossmatched, number of units transfused, and timing of transfusion.

Blood utilization indices are described as follows:

1) Crossmatch to transfusion ratio $(\mathrm{C} / \mathrm{T}$ ratio $)=$ number of units crossmatched/number of units transfused. A ratio of 2.0 and below is considered appropriate blood usage. $\mathrm{C} / \mathrm{T}$ ratio is an important national quality indicator that is used to gauge the appropriate use of services offered by the transfusion laboratory service. High $\mathrm{C} / \mathrm{T}$ ratio implies that crossmatches were performed unnecessarily when a Group-Screen and Hold
(GSH) would have sufficed. Our institution has adopted a ratio of greater than 2.0 as indicator for significant unutilized blood usage

2) Transfusion probability $(\% \mathrm{~T})=$ number of patients transfused/number of patients crossmatched $\times 100$. A value of $30 \%$ and above was considered indicative of significant blood usage

3) Transfusion index (TI) = number of units transfused/number of patients crossmatched. A value of 0.5 or more was considered indicative of significant blood utilization

4) Maximal Surgical Blood Order Schedule $(\mathrm{MSBOS})=1.5 \times \mathrm{TI}$

These are the cases and categorization of procedure done in whom blood was routinely requested preoperatively in our institution (Table 1).

\section{Exclusion criteria}

The exclusion criteria are as follows:

1.) Patients who underwent major trauma, orthopedic (i.e., amputation), and cardiac surgeries were excluded because these were patients who need liberal blood transfusion

2.) Patients who underwent major gynecologic and obstetric procedures

3.) Patients who needed pre-operative blood transfusion (with hemoglobin less than 10 or hematocrit less than 30)

4.) Patients who had ongoing blood losses in spite of adequate hematologic parameter (hemoglobin more than 10 or hematocrit more than 30 )

It is inappropriate to suggest a maximum surgical blood order to procedures relating to trauma, treatment of gastrointestinal hemorrhage, and major orthopedic procedures in children because they were associated with substantial post-operative hemorrhage.

\section{Data collection}

Data collected included the number of blood units crossmatched and transfused and the number of patients crossmatched and transfused. The data was used in the computation of the blood transfusion indices $(\mathrm{C} / \mathrm{T}$ ratio, $\% \mathrm{~T}$, and TI; see Section of Operational Terms). This will be categorized in the different surgical procedures pertinent to the age of the child. Lastly, the MSBOS was calculated in the different surgical procedures in the pediatric patient categorized by different age groups.

\section{Results}

During the 3-year study period, a total of 5314 cases were performed by the Division of Pediatric Surgery. 
Table 1 Cases and procedures where blood is requested pre-operatively in the Philippine Children's Medical Center

\begin{tabular}{ll}
\hline Cases & Procedure \\
\hline Major abdominal surgery & $\begin{array}{l}\text { Surgery for intestinal atresia, congenital diaphragmatic hernia repair, Ladd's procedure, } \\
\text { intussuception, bowel resection, adhesiolysis }\end{array}$ \\
$\begin{array}{l}\text { Major thoracic surgery } \\
\text { Surgery for abdominal wall defects }\end{array}$ & $\begin{array}{l}\text { Temporary silo placement, primary repair, staged repair } \\
\text { Head and neck surgery }\end{array}$ \\
Colorectal surgery & Pull through for anorectal anomalies and Hirschsprung's disease \\
Surgery for solid tumors & Excision of intraabdominal, retroperitoneal and sacrococcygeal tumors \\
Ostomies & Creation and closure of ostomy \\
Upper gastrointestinal Surgery & Esophageal replacement, esophageal, gastric and duodenal resection \\
Hepatobiliary & Surgery for biliary atresia, choledochal cyst, and biliary lithiasis \\
Genitourinary & Bladder reconstruction, renal surgery, repair of hypospadias \\
Appendectomy & Uncomplicated and complicated \\
\hline
\end{tabular}

Among this, 935 patients were identified and satisfied the inclusion and exclusion criteria. Of the 935 patients, a total of 1035 units of pRBC (packed RBC) were requested. Out of the 1035 units requested and crossmatched, only 405 units were transfused. This study revealed that the utilization rate was only $39.1 \%$ of the crossmatched blood. Of the total number of cases, $81.7 \%$ are done as elective cases.

The data collected was presented as blood utilization indices (see the "Methods" section) among procedures commonly performed at our institution (Table 1).

By eliminating $\mathrm{C} / \mathrm{T}$ ratio of procedures (highlighted in asterisk ":") that are more than 2.0, the calculated MSBOS can suggest the number of units crossmatched needed for procedures in which blood is commonly utilized (Table 2). The indices calculated in among the different procedures showed that in other procedures that have blood commonly crossmatched but infrequently transfused, a type and screen (T\&S) can be ordered in these procedures. Thus, a type screen for blood products can be requested for procedures not included in this new blood request policy. Only procedures involving major abdominal, major thoracic, abdominal wall defects, and hepatobiliary surgery require only 1 unit of pre-operative crossmatched blood.

\section{Discussion}

Friedman advocated the use of MSBOS with the goal to make pre-operative blood orders more closely coincide with the number of units of blood which will actually be transfused to patients undergoing surgical procedures [8]. But their study was limited to patients more than 18 years old. Pediatric patients were excluded because of the variable blood product requirements, which in children are influenced by blood volumes.

Currently, our institution is working on a blood transfusion policy that leads to efficient blood usage. Schmotzer et al. in 2010 reviewed their policy in which they

Table 2 Blood utilization indices among pediatric surgical patients in the Philippine Children's Medical Center

\begin{tabular}{|c|c|c|c|c|c|c|c|c|}
\hline \multirow[t]{2}{*}{ Procedure } & \multicolumn{2}{|c|}{ Number of units } & \multicolumn{2}{|c|}{ Number of patients } & \multirow{2}{*}{$\begin{array}{l}\mathrm{C} / \mathrm{T} \\
\text { ratio }\end{array}$} & \multirow[t]{2}{*}{$\% \mathrm{~T}$} & \multirow[t]{2}{*}{$\mathrm{Tl}$} & \multirow[t]{2}{*}{ MSBOS } \\
\hline & Crossmatched & Transfused & Crossmatched & Transfused & & & & \\
\hline Major abdominal surgery & 132 & 79 & 127 & 74 & 1.67 & 58.3 & 0.62 & 0.93 \\
\hline Major thoracic surgery & 54 & 31 & 45 & 29 & 1.74 & 64.4 & 0.69 & 1.03 \\
\hline Surgery for abdominal wall defects & 43 & 28 & 42 & 27 & 1.5 & 64.3 & 0.63 & 0.94 \\
\hline Head and neck surgery & 27 & 6 & 24 & 6 & $4.5^{*}$ & 25.0 & 0.22 & 0.33 \\
\hline Colorectal Surgery & 216 & 66 & 207 & 53 & $3.27^{*}$ & 25.6 & 0.32 & 0.48 \\
\hline Surgery for Solid tumors & 137 & 67 & 93 & 62 & $2.04^{*}$ & 66.7 & 0.72 & 1.08 \\
\hline Ostomy creation and closure of ostomy & 235 & 60 & 229 & 58 & $3.92^{*}$ & 25.3 & 0.26 & 0.39 \\
\hline Upper Gastrointestinal Surgery & 50 & 25 & 42 & 22 & 2.0 & 52.3 & 0.59 & 0.89 \\
\hline Hepatobiliary & 53 & 23 & 46 & 19 & $2.03^{*}$ & 41.3 & 0.50 & 0.75 \\
\hline Surgery for genitourinary abnormalities & 55 & 17 & 47 & 17 & $3.24^{*}$ & 36.2 & 0.36 & 0.54 \\
\hline Appendectomy & 33 & 3 & 33 & 3 & $11.0^{*}$ & 9.09 & 0.09 & 0.14 \\
\hline
\end{tabular}

${ }^{*} \mathrm{C} / \mathrm{T}$ ratio of more than 2.0 indicates unnecessary blood ordering 
implemented an evidence-based procedure-specific RBC preparation to each type of surgery in the pediatric age group [9]. In their review, they used the preparation-totransfusion ratio (P:T) instead of $\mathrm{C}: \mathrm{T}$, the $\mathrm{P}: \mathrm{T}$ ratio included uncrossmatched $\mathrm{RBC}$ aliquot in contrast to the C:T. The inclusion of uncrossmatched may not reflect current peri-operative blood ordering practices. They also used MSBOS guidelines, but their model used blood volumes that are specific to each surgical patient instead of the number of units transfused. In their model for each procedure, the volume used in each patient was calculated by subtracting the volume of blood returned to the blood bank from the volume issued by the blood bank to the patient. The procedure-specific volumebased guidelines developed in this system serve as a good estimate to the actual blood usage for each patient. Their results did not differentiate the actual blood usage of each type of procedure but rather estimate the blood volume to each type of surgical specialty (i.e., general surgery and orthopedic surgery). Surgical procedures were categorized into different specialties, eliminating the essence of procedure-specific blood transfusion requirements.

In our proposed model, the total unit used refers to normal units (250 cc per unit) and blood aliquots (a unit that has been divided into 2 or 3 smaller units). Understandably, the number of RBC units transfused may not accurately reflect total volume transfused or volume transfused in milliliter per kilogram. Although we can extract patient weight, we cannot extract the actual blood volume transfused as was previously done by the group of Schmotzer from our database. Instead, some aspects of our data were similar to the group by Keung et al. done in a tertiary Australian pediatric center [10].. They have used units of blood instead of volume on RBC transfusion. And they have described the percentage of transfusion specific to each procedure.

Some authors argue that MSBOS is of limited use. Palmer et al. [11] used and compared a patient-specific blood ordering system (PSBOS) in predicting who will receive transfusion than using a MSBOS. They have studied a formula to predict blood transfusion among adult patients undergoing elective surgery using starting hematocrit prior to surgery, estimated blood volume, anticipated blood loss, and predicted hematocrit at the end of the surgery. Their results showed the PSBOS has a high specificity at $93 \%$ but only sensitive at $41 \%$. The PSBOS takes into account perceived blood loss as judged by the operating surgeon and analyzes pre-op and post-op hematocrit, which theoretically can estimate actual blood usage. The low sensitivity as they reported limits its clinical use.

One of the limitations of the study was to exclude trauma patients; it may not be necessarily be true that there is no excessive blood ordering among this subgroup of patients. Surgeons and anesthesiologist currently advocate liberal transfusion policies in the trauma, but this practice can still change soon especially if we audit and analyze our results among trauma patients in the future.

The type and screen ( $\mathrm{T}$ and $\mathrm{S}$ ) approach is commonly accepted worldwide which is applied to cases where the transfusion probability is low $[1,12]$. The $\mathrm{T}$ and $\mathrm{S}$ approach can lead to a safe, effective and economic solution to ordering of blood especially to a resource-poor setting like ours. And from our results, we can adopt this policy and perform type and screen to procedures with less chance of blood transfusion.

There have been a few reports on the use of MSBOS in pediatric patients. Our series hopes to establish a baseline model as a way to improve blood utilization especially in resource-limited areas catering to the pediatric population. Our center handles various cases and has a different case mix compared to other pediatric centers. Our data is a reflection of the majority of cases handled in different institutions and thus can be extrapolated or compared to assess the blood usage in each pediatric procedure. Lastly, our data examined only RBC use and not anymore plasma and platelet. In the review of our data and matched to our inclusion and exclusion criteria, we have found that patients needing peri-operative platelet or plasma transfusion were patients with ongoing hemorrhage or major medical co-morbidities. This limits our analysis to the usage of RBC in our setting. Usage patterns of these other products would also be of interest and we can include these in our analysis in the future.

\section{Conclusion}

Our results show that only $39.1 \%$ of crossmatched blood is utilized; this is a reflection of inefficient blood usage practice. The computed blood indices $(\mathrm{C} / \mathrm{T}$ ratio, \% $\mathrm{T}$, and TI) among different procedures in different age group indicates that there are procedures where routine crossmatching of requested blood may be eliminated. The creation of MSBOS based on our own's institution data could further improve our current blood requisition practice. The MSBOS which is largely applied to adult population long with other blood utilization indices can be applied to pediatric blood usage. And our data shows that the majority of elective pediatric surgical procedures routine crossmatch is not necessary which is proven by our underutilization of requested blood products. Developing our own institution-based blood ordering policy which limits excessive blood ordering can reduce unnecessary compatibility testing, returning of unused blood and wastage due to outdating.

\section{Abbreviations}

\%T: Transfusion probability; $\mathrm{C} / \mathrm{T}$ ratio: Crossmatch to transfusion ratio; MSBOS: Maximal Surgical Blood Order Schedule; $\mathrm{P} / \mathrm{T}$ ratio: Patient to 
transfusion ratio; pRBC: Packed red blood cells; T \& S: Type and screen; TI: Transfusion index

\section{Acknowledgements}

Not applicable

\section{Authors' contributions}

JPG contributed to the conceptualization, protocol preparation, data collection, data analysis, and manuscript writing and editing. LLR and MBG contributed to the protocol preparation, data analysis, and manuscript writing. All authors have read and approved the final manuscript.

\section{Funding}

The article received no funding.

\section{Availability of data and materials}

The data is kept confidential and is in place within the records of the Division of Pediatric Surgery, Philippine Children's Medical Center.

\section{Ethics approval and consent to participate}

The article was approved by the ethical and technical review committee of the Philippine Children's Medical Center with protocol title PCMC IRB-EC 2017-023. Consent from the participants was not required because this study was retrospective in nature.

\section{Consent for publication}

Not applicable.

\section{Competing interests}

The authors declare that they have no competing interests.

Received: 1 October 2019 Accepted: 29 October 2019

Published online: 28 November 2019

\section{References}

1. Belayneh T, Messele G, Abdissa Z, Tegene B. Blood requisition and utilization practice in surgical patients at university of gondar hospital, northwest ethiopia. J Blood Transfus. 2013;2013:5. Article ID 758910. https:// doi.org/10.1155/2013/758910.

2. Hall TC, Pattenden C, Hollobone C, Pollard C, Dennison AR. Blood transfusion policies in elective general surgery: how to optimise crossmatch-to-transfusion ratios. Transfus Med Hemother. 2013;40(1):27-31.

3. Sowayan SA. Use of blood in elective surgery; an area of wasted hospital resource. Ann Saudi Med. 1994;14(4):326-8.

4. Mwambungu A, Siulapwa N, Mugala D, Chishimba M. Analysis of blood cross match ordering practice in surgical patients at Ndola Central Hospital. Int J Healthcare Sci. 2015;3(1):278-84.

5. Soomro R, Javed MR, Ali SA. Arrangements and use of blood in elective surgical procedures. Prof Med J. 2011;18(2):212-4.

6. Ballian N, Weisensel N, Rajamanickam V, Foley EF, Heise CP, Harms BA. Comparable post-operative morbidity and mortality after laparoscopic and open emergent restorative colectomy; outcomes from the ACS NSQIP. World J Surg. 2012;36:2488-96.

7. Vignali A, Ghirardelli L, di Palo S, Orsenigo E, Staudcher C. Laparoscopic treatment of adavanced colonic cancer; a case matched control with open surgery. Color Dis. 2013;13:944-8.

8. Friedman BA. An analysis of surgical blood use in United States hospitals with application to the maximum surgical blood order schedule. Transfusion. 1979;19(3):268-78.

9. Schmotzer CL, Brown AE, Roth S, Johnson J, Ines-Castillejo M, Reisner A, Hillyer CD, Josephson CD. Procedure-specific preoperative red blood cell preparation and utilization management in pediatric surgical patients. Transfusion. 2010;50(4):861-7.

10. Keung CY, Smith KR, Savoia HF, Davidson AJ. An audit of transfusion of red blood cell units in pediatric anesthesia. Pediatr Anesth. 2009;19(4):320-8.

11. Palmer T, Wahr JA, O'reilly M, Greenfield MLV. Reducing unnecessary crossmatching: a patient-specific blood ordering system is more accurate in predicting who will receive a blood transfusion than the maximum blood ordering system. Anesth Analg. 2003;96(2):369-75.

12. Gupta PK, Kumar H, Diwan RN. Blood ordering strategies in the armed forces-a proposal. Med J Armed Forces India. 2003;59(4):302.

\section{Publisher's Note}

Springer Nature remains neutral with regard to jurisdictional claims in published maps and institutional affiliations.

\section{Submit your manuscript to a SpringerOpen ${ }^{\circ}$ journal and benefit from:}

- Convenient online submission

- Rigorous peer review

- Open access: articles freely available online

- High visibility within the field

Retaining the copyright to your article

Submit your next manuscript at $\boldsymbol{\nabla}$ springeropen.com 